\title{
Special section on support technology and architecture for networked and distributed applications in big data era
}

\author{
Kin Fun Li ${ }^{1}$. Wendy Rahayu' ${ }^{2}$
}

Published online: 17 April 2015

(C) Springer Science+Business Media New York 2015

Advanced networking technology has grown rapidly and its applications have experienced an explosive growth. The networking revolution is transforming the way people live, work, play, and interact with each other, and impacting the way businesses, organizations, and governments operate. This special issue focuses on areas related to computing support technology, architecture and systems for big data applications, including both theoretical and practical aspects of supercomputing, algorithms, and performance evaluation.

Papers accepted in this special issue include submissions from a general call as well as extended versions of selected papers presented at the 27th IEEE International Conference on Advanced Information Networking (IEEE AINA-2013) held on March 25-28, 2013, in Barcelona, Spain. AINA-2013 received 567 submissions and has a $27 \%$ acceptance rate.

The first two papers are security related. Xhafa et al. propose an attributed-based encryption scheme for personal health record in cloud environments, while Palmieri et al. investigate denial of service attacks aimed at consuming a large amount of energy in cloud-based infrastructures.

The next three papers address energy consumption: Doulikun et al. discuss a dynamic energy aware algorithm in a cluster of servers; Lin et al. consider policies for storing and replicating intermediate data in clusters to increase reliability and lower energy consumption; and Abid et al. propose a hybrid method to improve aggregation gain and energy consumption for time-constrained wireless sensor networks.

\footnotetext{
Kin Fun Li

kinli@uvic.ca

1 University of Victoria, Victoria, Canada

2 La Trobe University, Melbourne, Australia
} 
Four data and task movement papers wrap up this special issue. Dhurandher et al. predict locations of message passing in opportunistic networks to enhance routing. Stenico and Ling model network traffic based on binomial multiplicative cascades. Wang et al. attempt to improve massive data transfer in Hadoop clusters. Finally, Pop et al. model scheduling of aperiodic tasks with deadline within inter-Cloud environments.

We would like to thank all the authors and all the reviewers for their hard work to make this special issue possible. In particular, we would like to thank Editor-in-Chief of The Journal of Supercomputing, Hamid R. Arabnia, for his advice and support. 